\section{Demystifying peer review}

\section{Peer review is an essential aspect of the publishing process; journals and the research community have a shared interest in it being a constructive process.}

Peer review, at its best, should aim to provide authors and editors with rigorous and constructive feedback resulting in an improved study. This system, like any, has its deficiencies and finding productive ways to improve it is important. For example, authors are often frustrated by a seemingly opaque process, and providing more transparency into how papers are selected for publication is a goal for this journal. To this end, Nature Cell Biology editors give talks at conferences and research centres to inform authors and referees of the key factors that guide editorial decisions and the referee selection process.

Commonly in the biological sciences, professional or academic editors shepherd papers through a single-blind peer review process. A group of stem cell researchers have recently advocated greater transparency in the process, specifically requesting that journals release referee reports and the correspondence between authors and editors for published manuscripts (http://www.eurostemcell.org/commentanalysis/peer-review). The EMBO Journal has, for example, been publishing this information since 2009. Informal discussions with scientists who work in diverse areas covered by the journal has revealed mixed support for this practise. One issue is that the quality of reviews may be adversely affected if reviewers thought that their identities may be revealed through their reports. This is a particular concern in contentious areas that are dominated by divergent perspectives from a small group of researchers. It is also difficult to determine the validity of referee comments without at least knowing the expertise of the referees and ultimately, these disclosures might be more illuminating in the case of difficult decisions involving rejected manuscripts. Others argue that this practise would be educational for new PIs, graduate students and postdocs who may have less direct experience of the editorial and peerreview process, and that referees may provide more constructive reports when faced with the prospect of having their reports made public. At this journal, several measures are in place to promote a balanced review process. For example, our reviewers receive a copy of each other's comments postdecision, giving them the opportunity to provide feedback on other reports if warranted. This can be beneficial when manuscripts receive strikingly polarized feedback from referees with common expertise. We also strongly encourage reviewers to include all information relevant to the assessment of a manuscript in the comments to the author. Editorial decisions often involve further discussions with authors and referees, by phone, e-mail or at conferences and we may involve additional experts to arbitrate between divergent referee inputs. The complexity of this dialogue between editors, authors and referees is difficult to fully capture in referee reports and decision letters alone.

Post-review rejection can be frustrating for the author, particularly when significant effort was made to address referee comments. Authors at Nature research journals can transfer manuscripts, along with the complete reports and the referee identities, between Nature journals, thereby making the publication process more efficient for authors and referees. The neuroscience community has gone a step further; the Neuroscience
Peer Review Consortium, formed in January 2008, is a collection of about 30 neuroscience journals who have agreed to share referee reports and referee identities with each other, following permission from the referee. A potential benefit of this approach is that recycling reports between journals may help reduce the workload of referees who are reviewing multiple manuscripts for different journals.

Although there is clearly room for improvement, the current system is not broken. The onus is on journals and editors to ensure timely, rigorous and fair peer review. A diverse pool of referees to guard against overrepresentation of a narrow viewpoint is essential. Drawing on a large referee bank can also help reduce delays and avoid referee 'burnout' from reviewing multiple manuscripts for a given journal. Vetting referees for quality and consistency is crucial. Ensuring that referees are aware of our guidelines regarding disclosures of conflict of interest and adhere to them is also important. Authors frequently bemoan the often exhaustive list of issues that must be addressed prior to publication and rightly expect editors to prioritize referee requests. At this journal, editors actively mediate the discussion between authors and referees to not only identify areas that must be strengthened but also to overrule on points that may be beyond the scope of a given study.

The peer review process will be inherently subjective as it relies on the opinions of selected experts in the field. Thus, seeking broader input from the community on published papers as we routinely do at conferences is highly valuable and helps inform future decisions.

We encourage our readers to provide us with direct feedback on the issues discussed here, along with suggestions for further improvements.

\section{Nature Communications}

\section{A new Nature research journal for the biological, physical and chemical sciences.}

As this issue goes to press, Nature Communications, a new Nature journal dedicated to primary research in the biological, physical and chemical sciences will have launched. The journal aims to publish papers that provide a significant advance for specialists within a discipline but that do not have the broader scientific impact of papers published in Nature and the Nature research journals. Nature Communications will be an online-only journal with continuous online publication thus enabling rapid dissemination of primary research. Authors may also choose to make their published work open access.

Like all other Nature journals, manuscripts submitted to Nature Communications will be subject to rigorous peer-review. Unlike other Nature journals, Nature Communications has an editorial advisory board that the editors may consult with. Manuscripts rejected from Nature Cell Biology - either before or after review - may be transferred to Nature Communications using a link provided in the decision letter. In the case of reviewed manuscripts, Nature Communications will receive referee reports and their identities through the automated transfer process. Nature Communications is editorially independent and the editors will determine whether the transferred manuscript is suitable for further consideration. The editors will take into account the transferred referee reports when reaching a decision but they may also choose to consult with independent experts. 